\title{
CAPÍTULO 11: DESENVOLVIMENTO E CARACTERIZAÇÃO MICROBIOLÓGICA DE RICOTA CONDIMENTADA COM ORÉGANO ELABORADA COM LEITE DE CABRA
}

\section{CHAPTER 11: DEVELOPMENT AND MICROBIOLOGICAL AND PHYSICAL AND CHEMICAL CHARACTERIZATION OF RICOTA SPICED WITH ORGAN}

\author{
Antonio Alef Pereira de Oliveira ${ }^{1}$; Layane Rosa da Silva ${ }^{2}$; Aliou Toro Lafia ${ }^{3}$; José Douglas Bernardino \\ Domingos $^{4}$; Fabiana Augusta Santiago Beltrão ${ }^{5}$
}

\begin{abstract}
Resumo
A ricota é um produto de origem italiana fabricada por meio de precipitação ácida para separação das proteínas do soro do leite. A ricota pode ser consumida ao natural, com ou sem sal, e saboreada com mel, açúcar ou frutas, ou ainda condimentada com alho, salsa, orégano e outros. Objetivou-se com este trabalho processar e avaliar ricota adicionada de diferentes concentrações alho e orégano e avaliar sua estabilidade microbiológica. Foram elaboradas quatro formulações: ricota tradicional (T1); ricota condimentada com orégano (T2); ricota condimentada com alho (T3) e ricota condimentada com alho e orégano (T4), as quais foram submetidas à análise microbiológica após 10 dias de armazenamento refrigerado $\left(5 \pm 2{ }^{\circ} \mathrm{C}\right)$. Os resultados das análises microbiológicas indicaram que todas as amostras se encontravam dentro dos padrões estabelecidos pela legislação vigente para queijos de alta umidade.
\end{abstract}

Palavras-Chaves: Ricota, bromatologia, condimentos.

\begin{abstract}
Ricotta is a product of Italian origin manufactured using acid precipitation to separate whey proteins. The ricotta can be eaten in its natural state, with or without salt, and savored with honey, sugar or fruit, or seasoned with garlic, parsley, oregano and others. The objective of this work was to process and evaluate ricotta added with different concentrations of garlic and oregano and to evaluate its microbiological stability. Four formulations were prepared: traditional ricotta (T1); ricotta seasoned with oregano (T2); ricotta seasoned with garlic (T3) and ricotta seasoned with garlic and oregano (T4), which were submitted to microbiological analysis after 10 days of cold storage $\left(5 \pm 2^{\circ} \mathrm{C}\right)$.
\end{abstract}

Keywords: Ricotta, bromatology, condiments.

\section{Introdução}

A partir do soro do leite pode ser elaborado o queijo ricota, um produto de origem italiana, e considerado um dos queijos mais magros, devido ao teor reduzido de lipídeos e de calorias em sua composição (SPANU et al., 2018). A ricota fresca é definida como

\footnotetext{
${ }^{1}$ Bacharelado em Agroindústria, Universidade Federal da Paraíba/UFPB, aleffjoe@ gmail.com

${ }^{2}$ Bacharelado em Agroindústria, Universidade Federal da Paraíba/UFPB, layanerossa@ gmail.com

${ }^{3}$ Mestrando em Tecnologia Agroalimentar, Universidade Federal da Paraíba/UFPB, zime1990@ gmail.com

4 Bacharelado Nutrição e Dietética, Universidade Federal de Campina Grande/UFCG, douglas94nc@gmail.com

${ }^{5}$ Doutora em Biotecnologia, Universidade Federal da Paraíba/UFPB, fabs.15@ hotmail.com
} 
o queijo obtido pela precipitação ácida a quente de proteínas do soro de leite, com adição de leite, até vinte por cento do seu volume, devendo apresentar consistência mole e coloração branca ou creme, formato cilíndrico e peso variando entre $300 \mathrm{~g} \mathrm{e} 1 \mathrm{~kg}$ (BRASIL, 2017).

O elevado consumo de ricota se deve pelo seu ao alto valor proteico, baixo teor de sódio e gorduras. Por estas razões é amplamente utilizada pelos consumidores adeptos de uma dieta saudável (ROSAS et al., 2016; VALADÃO, 2015). A ricota ainda apresenta um sabor acentuado e ligeiramente agradável, que pode ser consumido ao natural, com ou sem sal, condimentada ou defumada (BRASIL, 2017). O uso de condimentos e especiarias em alimentos tem crescido largamente, pois além de conferirem sabor, auxiliam na digestão, aprimoram as características sensoriais do alimento e possuem propriedades terapêuticas (SEDLMAIER et al., 2017).

$\mathrm{O}$ orégano é uma planta aromática e medicinal Origanum vulgare pertence à família Lamiaceae. Esta erva aromática é vulgarmente chamada de orégão vulgar ou manjerona selvagem e são utilizadas várias partes da sua estrutura física para diversos fins, pois os seus metabolitos secundários conferem-lhe algumas propriedades tanto condimentares como medicinais, é considerada uma planta tónica e tem uma grande diversidade de propriedades medicinais, sendo as propriedades digestivas e expetorantes as que mais se enfatizam (Norman, 2004).

O alho (Allium sativum L.) é uma hortaliça rica em amido e substâncias aromáticas de alto valor condimentar e possui ação fitoterápica com diversas propriedades farmacológicas. Uma das espécies cultivadas mais antigas começou a ser plantado há mais de 5.000 anos pelos hindus, árabes e egípcios. Nesse contexto, se torna relevante o uso de novos ingredientes naturais em formulações de ricota como o alho e o orégano. Ainda, verifica-se a importância de prolongar a vida útil e agregar um novo sabor característico ao produto, configurando-se como uma nova e viável opção de consumo (Norman, 2004).

Mesmo dotado de excelentes características nutricionais, o leite de cabra não é visto como um bom atraente pelos consumidores, por isso é de suma importância que sua divulgação e marketing possibilitem mostrar sua qualidade, devendo ser feito junto às associações e/ou cooperativas de produtores, para que possa discutir e propor políticas de fomento a esta atividade por meio de uma produção sustentável. Bem como a realização de mais pesquisas que visem disponibilizar aos produtores informações com fácil entendimento e aplicabilidade, mediante o tipo sistema de produção adotado (Cavalcanti, 
2014).

Diante do que foi abordado em termos de pesquisa e em face de desenvolvimento socioeconômico de regiões que atuam com a caprinocultura leiteira, o leite de cabra ainda é um produto pouco estudado no tange o seguimento e aplicação ao mercado, fazendo com que a ricota se torne apenas um derivado opcional para aplicação de beneficiamento do leite, dessa forma, para o aproveitamento do soro de queijos (Cutrim. 2017).

Nesse contexto, busca-se através do desenvolvimento e caracterização de ricota de leite de cabra, com adição de orégano, agregando valor nutricional, tornando uma nova opção de derivado lácteo, gerando o estimulo a produção de produtos lácteos com o leite de cabra principalmente para pequenos produtores, com pensamento sustentável, evitando desperdício do leite e estimulando a produção e consequentemente a renda, o que configura a principal motivação para a elaboração deste trabalho.

\section{Material e Métodos}

As análises microbiológicas foram realizadas segundo as recomendações metodológicas sendo pesquisados os seguintes microrganismos: coliformes a $35^{\circ} \mathrm{C}$; Estafilococus coagulase positiva; bolores e Salmonella. As ricotas foram elaboradas no laboratório de laticínios da Universidade Federal da Paraíba, seguindo rigorosamente todas as boas práticas de manipulação e de fabricação de alimentos. Os ingredientes foram adquiridos da feira local da cidade de Bananeiras-PB, com exceção do leite de cabra que foi do laboratório de ovinos e caprinos da Universidade Federal da Paraíba.

Para elaboração foram utilizados os seguintes ingredientes soro do leite, orégano, alho e vinagre. Foram elaboradas quatro formulações de ricota, tradicional (T1); ricota condimentada com orégano (T2); ricota condimentada com alho (T3) e ricota condimentada com alho e orégano (T4).

$\mathrm{O}$ processamento da ricota aconteceu da seguinte forma como mostra o fluxograma abaixo. 


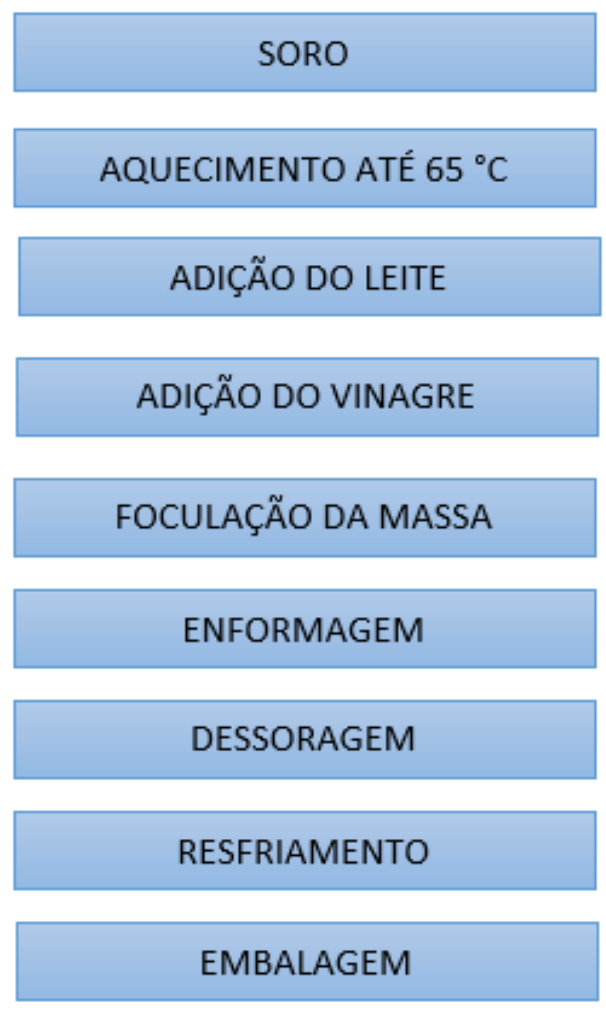

Inicialmente o soro do leite de cabra foi colocado em um recipiente levado ao fogo e agitado de forma lenta e constante até atingir $65^{\circ} \mathrm{C}$, sempre controlando a temperatura com termômetro. Depois foi feito a adição do leite e os condimentos assim que atingiu a temperatura de $65{ }^{\circ} \mathrm{C}$, adicionando lentamente de 5 a $8 \%$ de leite desnatado, levando-se em consideração o volume inicial do soro, ou seja, 5 a 8 litros de leite para cada 100 litros de soro. Após a adição do leite e os condimentos, continua-se a agitação até atingir $85^{\circ} \mathrm{C}$. Nesse momento é preciso ir adicionado o vinagre branco lentamente na proporção de 0,1 com relação ao volume do leite e de soro, ou seja, $100 \mathrm{ml}$ de vinagre branco para cada 100 litros da mistura soro/leite, mexendo a mistura por 3 minutos para melhor incorporação. Após a incorporação do vinagre, é possível observar observa-se a formação de flocos na superfície da mistura, nesse momento deve-se interromper a agitação, e controlar a temperatura até atingir $95{ }^{\circ} \mathrm{C}$. Em seguida, foi retirada a fonte de calor e deixada a mistura em repouso por 30 minutos para que acontecesse o resfriamento. Depois desse descanso é possível observa na superfície da mistura a formação de uma massa branca flutuante, resultando na Ricota. Essa massa flutuante foi retirada cuidadosamente, com o auxílio de uma peneira, evitando que se quebre os flocos. Em seguida colocadas e formas para assim a massa ficar em repouso e acontecer a dessoragem. Após ficar em temperatura ambiente, as ricotas ainda nas formas foram levadas para resfriamento a temperatura de 6 a $10{ }^{\circ} \mathrm{C}$ por um período de 24 horas. 
A utilização do leite desnatado deve-se principalmente ao fato da Ricota ser um queijo para pessoas em dieta de restrição alimentar ou que necessitem de alimentos de fácil digestibilidade. Após 30 minutos de repouso, foi retirada com cuidado a massa floculada, com auxílio de uma peneira, evitando-se quebrar os flocos. A adição de orégano e alho foi de $10 \mathrm{~g}$ para formulação de ricota condimentada com orégano (T2); ricota condimentada com alho (T3) e ricota condimentada com alho e orégano (T4). A ricota de (T1) não foi adicionado nenhum tipo de condimento, permanecendo no modo tradicional.

Para as análises microbiológicas foram seguidas de acordo com a RDC n ${ }^{\circ} 12$ de 2 de janeiro de 2001, que estabelece os limites microbiológicos para queijos com alto teor de água, temperados, condimentados ou adicionados de ervas ou outros ingredientes é de Coliformes a $45^{\circ} \mathrm{C} / \mathrm{g} 10^{2}$, Staphylococcus coagulase positiva/g $10^{3}$ e Salmonella $\mathrm{sp} / 25 \mathrm{~g}$ Ausência.

Para a determinação de Estafilococos coagulase positiva, foi transferido com o auxílio de uma pipeta $1 \mathrm{ml}$ do diluente para as placas de petri, sendo em seguidas incubadas em estufa a $35^{\circ} \mathrm{C}$ por 24 horas, após este período foi verificado se houve presença ou ausência para este microrganismo. A partir das diluições sucessivas $10^{-1}, 10^{-}$ 2 e $10^{-3}$ transfere $1 \mathrm{ml}$ da amostra para a placa de petri e depois transferidos o meio Aga Batata Dextrose (BDA) aguarda-se a solidificação do meio e incuba a $25^{\circ} \mathrm{C}$ de 3 a 5 dias.

Para a determinação de salmonela inicialmente foi realizado um plaqueamento seletivo diferencial, para isso foi homogeneizado o tubo de enriquecimento e retirado com uma alça de platina uma alçada do Caldo Tretationato e feita a estria em placas de Agar Entérico de Hectoem e Agar Xilose Lisina descarboxilado como também em Agar verde brilhante Agar Salmonela diferencial ou Agar Salmonella Shigela, após estes procedimentos as placas foram invertidas e incubadas a $35^{\circ} \mathrm{C}$ por 24 horas, logo após este período foi verificado se houve o desenvolvimento de colônias típicas de Salmonella. Para a confirmação das colônias típicas de Salmonella, com o auxílio de uma agulha de inoculação foi removido uma porção da massa das células, do centro da colônia típica e semeado nos tubos contendo Agar Lisina ferro e Agar Tríplice açúcar. Logo os tubos foram incubados a $35^{\circ} \mathrm{C}$ por 24 horas. Para o teste Bioquímico foi transferido com o auxílio de uma agulha através de uma picada a cultura de colônia típica de TSI para o meio e incubado a $35^{\circ} \mathrm{C}$ por 24 horas, após este período foi verificado se houve presença ou ausência de salmonela no produto. 


\section{Resultados e Discussão}

A Tabela 1 mostra os resultados de coliformes totais e termotolerantes (NMP/g), e enterobactérias (UFC/g) das ricotas tradicional e condimentadas com orégano e alho nos tempos de 1,7 e 21 dias de armazenamento refrigerado.

Tabela 1 - Resultados de coliformes totais e termotolerantes (NMP/g), e enterobactérias (UFC/g) das ricotas tradicional e condimentadas com orégano e alho

\begin{tabular}{llllll}
\hline Determinações & $\begin{array}{l}\text { Tempo de } \\
\text { armazenamento }\end{array}$ & RT & RO & RA & ROA \\
& 1 dia & 3,6 & 9,2 & 23 & 23 \\
\hline Coliformes totais $-35^{\circ} \mathrm{C}$ & 1 dias & $<3,0$ & 3,6 & 23 & $<3,0$ \\
& 21 dias & 240 & $<1.100$ & $<3,0$ & 290 \\
& 1 dia & $<3,0$ & $<3,0$ & 23 & $<3,0$ \\
Coliformes & 7 dias & $<3,0$ & 3,6 & 23 & $<3,0$ \\
termotolerantes $-45^{\circ} \mathrm{C}$ & 21 dias & 3,6 & $<3,0$ & $<3,0$ & $<3,0$ \\
$(\mathrm{NMP} / \mathrm{g})$ & 1 dia & $<10^{2}$ & $<10^{2}$ & $<10^{2}$ & $<10^{2}$ \\
& 7 dias & $<10^{2}$ & $<10^{2}$ & $3 \times 10^{2}$ & $10^{3}$ \\
Enterobactérias & 21 dias & $7,7 \times 10^{4}$ & $2,3 \times 10^{5}$ & $3,1 \times 10^{6}$ & $1,4 \times 10^{6}$ \\
\hline
\end{tabular}

Legenda: RT - ricota tradicional; RO - ricota condimentada com orégano; RG - ricota condimentada com alho; ROA - ricota condimentada com orégano e alho .

Os resultados de coliformes totais das ricotas tradicional e condimentadas, nos tempos 1 e 7 dias de armazenamento refrigerado, variaram de <3,0 a 23 NMP/g. Aos 21 dias, os resultados de coliformes totais da ricota condimentada com açafrão- da-terra (RT), da ricota condimentada com açafrão-da-terra e gengibre (RAO) e da ricota tradicional (RT) foram <1.100, 290 e $240 \mathrm{NMP} / \mathrm{g}$, respectivamente. Já a ricota condimentada com gengibre (RA) apresentou menor contaminação ( $<3,0$ NMP/g).

A legislação brasileira não determina limites de tolerância para este grupo de micro-organismos em ricota (BRASIL, 2001). Entretanto, por ser um alimento de vida de prateleira limitada e por apresentar elevada umidade e disponibilidade de nutrientes a ricota é considerada um alimento susceptível ao crescimento de bactérias deteriorantes e patogênicas. A presença de coliformes totais nas ricotas, mesmo que em baixas contagens, pode indicar falhas higiênicas ocorridas durante a manipulação do produto ou pós processamento do mesmo, ou até mesmo a má qualidade das matérias-primas utilizadas (VALIATTI et al., 2017).

No estudo de Sales et al. (2016), que avaliou 10 amostras de ricotas frescal 
adquiridas em supermercados do município de Curitiba - PR, foi verificada a presença de coliformes totais em $80 \%$ das amostras analisadas, com valores variando de 2 a 999 UFC/25 g. Os autores concluíram que estes resultados indicaram falhas higiênicas durante a produção e manipulação do produto.

Preis et al. (2015) verificaram que ricotas adicionadas de condimento composto por amido de milho, glutamato monossódico, sal, manjericão em flocos, sementes moídas de funcho, orégano desidratado pó/moído, pimenta preta moída, pimenta branca moída, cebola desidratada em pó, louro em pó, coentro moído/pó, açúcar, vegetais desidratados e corante natural de cúrcuma, apresentaram contagem inferior a $3 \mathrm{NMP} / \mathrm{g}$ de coliformes totais, enquanto a ricota padrão (sem especiarias) apresentou contagem de $23 \mathrm{NMP} / \mathrm{g}$. Os resultados encontrados pelos autores demonstraram a eficácia do condimento composto na inibição do crescimento de coliformes totais em ricotas.

Dessa forma, considerando o potencial antimicrobiano do orégano e do alho já relatado na literatura, pode-se atribuir a este condimento, na concentração testada neste estudo, um efeito inibidor do crescimento de coliformes totais nas ricotas ao longo dos 21 dias de armazenamento refrigerado (CUTRIM, 2017; SANTOS, 2015).

Os resultados de coliformes termotolerantes das ricotas tradicional e condimentadas, nos tempos 1, 7 e 21 dias de armazenamento refrigerado, variaram de $<3,0$ a $23 \mathrm{NMP} / \mathrm{g}$. Desta forma, todas as amostras, em todos os tempos avaliados, encontravam-se de acordo com a legislação vigente, a qual determina 5,0 x $10^{2} \mathrm{NMP} / \mathrm{g}$ de coliformes a $45^{\circ} \mathrm{C}$ como sendo o limite tolerável em queijos de muito alta umidade (BRASIL, 2001). Os resultados encontrados se assemelham aos obtidos por Andrade et al. (2014) que, ao avaliar ricota natural e ricota condimentada com alho, salsa, manjericão e ervas-finas, detectaram valores inferiores a 3,0 NMP/g em todas as amostras de ricota, nos tempos zero e 7 dias de armazenamento refrigerado a $10{ }^{\circ} \mathrm{C}$.

No primeiro tempo de análise das ricotas não foi observada a presença de enterobactérias em níveis detectáveis $\left(<10^{2} \mathrm{UFC} / \mathrm{g}\right)$. No entanto, após 7 dias de armazenamento, as ricotas condimentadas com orégano (RA) e orégano e alho (RAO) apresentaram contagens de $3 \times 10^{2}$ e $10^{3} \mathrm{UFC} / \mathrm{g}$, respectivamente. Após 21 dias, todas as amostras apresentaram resultados elevados de enterobactérias, variando de 7,7 x $10^{4}$ a $3,1 \times 10^{6} \mathrm{UFC} / \mathrm{g}$. Os resultados encontrados neste estudo indicam que o orégano e o alho, nas concentrações utilizadas, não apresentaram efeito inibidor sobre esse grupo microbiano ao longo do período de armazenamento. 
Embora a legislação brasileira não disponha de padrões para este grupo de microorganismos a sua avaliação é importante, pois muitas enterobactérias são utilizadas como indicadores biológicos de contaminação em água, alimentos e instrumentos. Este grupo inclui bactérias dos gêneros Escherichia, Shigella, Salmonella, Enterobacter, Klebsiella, Serratia, Proteus e outros. Alguns destes micro-organismos podem causar doenças, incluindo gastroenterites e infecções do trato urinário, como também podem produzir toxinas que causam intoxicações (NOGUEIRA et al., 2013; FREITAS et al., 2015).

No presente trabalho não foi observada a presença de estafilococos coagulase positiva nas ricotas tradicional e condimentadas em nenhum dos tempos avaliados $\left(<10^{2}\right.$ $\mathrm{UFC} / \mathrm{g}$ ). Portanto, todas as amostras encontravam-se de acordo com a legislação, a qual determina que para queijos de alta umidade elaborados por coagulação enzimática sem a ação de bactérias lácticas o limite tolerável de estafilococos coagulase positiva é 5,0 x $10^{2} \mathrm{UFC} / \mathrm{g}$ (BRASIL, 2001).

Em contrapartida, no estudo de Carrijo et al. (2011) foi detectada a presença de Staphylococcus spp. em todas as amostras de ricotas frescas comercializadas no município de Niterói, Rio de Janeiro, e estafilococos coagulase positiva em 50\% delas, classificando-as como impróprias para o consumo humano. A identificação de microorganismos do gênero Staphylococcus em leite e derivados é relevante pois este microorganismos produz uma toxina termorresistente, ou seja, que resiste ao tratamento térmico ao qual o leite é submetido. Esta toxina pode causar surtos de intoxicação estafilocócica (CAVALCANTI, 2014; CERESER et al., 2011).

No que diz respeito à Salmonella sp., todas as amostras analisadas, em todos os tempos de armazenamento refrigerado, exibiram ausência em $25 \mathrm{~g}$, apresentando-se em conformidade com a legislação vigente (BRASIL,2001).

\section{Conclusões}

As ricotas condimentadas demonstraram estabilidade microbiológica de acordo com os parâmetros estabelecidos pela legislação, estando aptas para o consumo. Diante do exposto, conclui-se que a utilização de condimentos combinados em ricotas é uma alternativa viável e promissora, uma vez que minimiza as alterações negativas nas características sensoriais da ricota ao mesmo tempo em agrega valor ao produto. 


\section{Referências}

ANDRADE, S. FLORESTA, P. M. M.; MARTINS, A. D. O.; MARTINS, M. P.;MARTINS, J. M. Avaliação das características físico-químicas, microbiológicas e sensoriais de ricotas em pasta condimentadas. VÉRTICES, Campos dos Goytacazes/RJ, v.16, n.1, p. 87-99, jan/abr. 2014.

ARRAIS, B. C. D. Desenvolvimento de ricota funcional: avaliação das características físico-químicas e microbiológicas do produto. 2015. 57f. Dissertação (Mestrado em Ciência e Tecnologia do Leite) - Universidade Norte do Paraná- UNOPAR, Londrina, 16. Marc, 2015.

ASQUI, M. G. P.Diseño del proceso de elaboración de queso riccota a partir del suero láctico de la quesera "El Sinche". 2017. 86f. Monografia (Graduação em Engenharia Química) - Escuela Superior Politécnica de Chimborazo, Riobamba Ecuador, 2017.

AZEVEDO, L. M.; FILHO, F. C. C.; LIMA, M. T.; SILVA, A. A.; et al. Vida de prateleirade ricota temperada. Revista Brasileira de Higiene e Sanidade Animal, Piauí, v. 10, n. 1, p. 1-8, jan-marc, 2016.

BALD, J. A.;VINCENZI, A.; GENNARI, A.; LEHN, D. N.; SOUZA, C. F. V.Características físico-químicas de soros de queijo e ricota produzidos no Vale do Taquari, RS. Revista Jovens Pesquisadores, Santa Cruz do Sul, v. 4, n. 3, p. 90- 99, 2014.

BARBOSA, E. D. Secagem do extrato aquoso de gengibre (Zingiber officinale Roscoe) pelo método de camada de espuma. 2016. 39f. Trabalho de Conclusão de Curso - Universidade Tecnológica Federal do Paraná, Campo Mourão, 2016.

BRASIL, Agência Nacional de Vigilância Sanitária, ANVISA, Resolução - CNNPA no 12, de 1978. Aprova as normas técnicas especiais, do Estado de São Paulo, revistas pela CNNPA, relativas a alimentos e bebidas, para efeito em todo território brasileiro, Diário oficial da União, 24 Jul. 1978.

BRASIL. Agência Nacional de Vigilância Sanitária. Resolução RDC $\mathrm{n}^{\circ} 12$, de 02/01/2001. Regulamento técnico sobre padrões microbiológicos para alimentos. Diário Oficial da República Federativa do Brasil. Brasília, DF, 2001.

BRASIL, Decreto $n^{\circ} 9.013$, de 29 de março de 2017, Regulamentada Lei no 7.889 , de 23 de novembro de 1989, que dispõem sobre o Regulamento da Inspeção Industrial e Sanitária de Produtos de Origem Animal. Diário Oficial da União, Brasília, DF, Ministério da Agricultura, Pecuária e Abastecimento, 3 out. 2017.

BRASIL, Ministério da Agricultura, do Abastecimento e da Reforma Agrária. Portaria No 146, de 7 de Março de 1996. Regulamento da Inspeção Industrial e Sanitária de Produtos de Origem Animal. Diário Oficial da União, Brasília, DF, Ministério da Agricultura, Pecuária e Abastecimento, 11 Mac.1996.

BRASIL. Ministério da Agricultura, Pecuária e Abastecimento. Instrução Normativa 
$\mathrm{n}^{\circ}$ 62, de 26 de agosto de 2003. Métodos Analíticos Oficiais para Análises Microbiológicas para o Controle de Produtos de Origem Animal e Água. Diário Oficial da União, Brasília, DF,18 Set, Seção 1, 2003.

BRASIL. Ministério da Saúde, Agência Nacional de Vigilância Sanitária. Resolução RDC n 276, de 22 de Setembro de 2005. Regulamento técnico para especiarias, temperos e molhos. Diário Oficial da República Federativa do Brasil. Brasília, DF, 23 set. 2005 .

CAJAS, A. X. T. Desarrollo de un condimento a base de vegetales deshidratados y especias bajo en sodio, utilizando cloruro de potasio como sustituto de sal. 2018. 88f. Monografia (Graduação em Química dos Alimentos) - Faculdade de Ciências Químicas, Quito, Fev, 2018.

CARRIJO, K. F.; PRAXEDES, C. I. N. S.; NOBRE, F. S. D.; DUARTE, M. T.; et al.Condimentos e especiarias empregados no processamento de alimentos: considerações a respeito de seu controle físico-químico. PUBVET, Londrina, v. 6, n. 26, Ed. 2013, p. 1-27, Art. 1417-1422, 2012.

CERESER, N. D.; JÚNIOR, O. D. R.; MARCHI, P. G. F.; SOUZA, V.; et al. Avaliaçãoda qualidade microbiológica da ricota comercializada em supermercados do estado de São Paulo. Embrapa Caprinos e Ovinos-Artigo em periódico indexado (ALICE), 2011.

CAVALCANTI, F. B. Avaliação microbiológica do queijo "tipo ricota" comercializados em supermercados de Campina Grande-PB. 2015. 29p. Monografia (Graduação em Química Industrial) - Universidade Estadual da Paraíba, Campina Grande - PB, 2014.

CUNHA, E. O. Análise comparativa da qualidade nutricional de ricotas tradicional, light e orgânica vendidas em João Pessoa. 2014. 35f. Monografia.

(Graduação em Nutrição) - Departamento de Nutrição da Universidade Federal da Paraíba- UFPB, João Pessoa, 2014.

CUTRIM, E. S. M. A avaliação antimicrobiana e antioxidante dos óleos essenciais de zingiber officinale Roscoe (gengibre) e Rosmarinus officinalis L. (alecrim) frente ás bactérias patogênicas. 2017. 69f. Monografia (Graduação em Química Industrial) Universidade Federal do Maranhão, São Luís, 2017.

DABAGUE, I. C. M.; DESCHAMPS, C.; MACHADO, M. P.; CÔCCO, L.

C.Rendimento do óleo essencial de Zingiberofficinale em resposta a diferentes processamentos e tempos de extração. Revista Acadêmica Ciência Agrár. Ambient., Curitiba, v. 11, Supl. 2, p. S163-S168, 2013.

DAMER, J. R. S.; MORRESCO, T. R.; WESCHENFELDER, S.

Qualidademicrobiológica de queijo ricota comercializado na região Noroeste do Rio Grande do Sul. Revista Brasileira de Ciência Veterinária, Rio Grande do Sul, Brasil, v. 22, n. 3-4, p. 216-219, jul/dez. 2015-2016.

DETONI, E.; GONÇALVES, L.A. Desenvolvimento de creme de ricota 
condimentado com tomate seco e manjericão. 2011. 40f. Monografia (Graduação em Tecnologia em Alimentos) - Universidade Tecnológica Federal do Paraná, Francisco Beltrão - PR, 2011.

DORNELLAS, F. C. Atividade antifúngica de Cúrcuma longa L.(Zingiberaceae) contra fungos deteriorantes de pães. 2016. 33f. Monografia (Graduação em Tecnologia de Alimentos) - Universidade Tecnológica Federal do Paraná - UTFP, Campo Mourão, 2016.

DUARTE, A. F. S.; MARTINS, A. L. C.; MIGUEL, M. D.; MIGUEL, O. G. O uso de plantas medicinais durante a gravidez e amamentação. Visão Acadêmica, Curitiba, v.18, n.4, p.126-139, out/dez, 2017.

FREITAS, M. P. Avaliação microbiológica de queijos artesanais produzidos na cidade de Taió, Santa Catarina. Revista Interdisciplinar Saúde Meio Ambient. v. 4, n. 2, p. 103 114, jul./dez. 2015.

HORBAN, M. A.; SILVA, A. A.; MAYDL, M. P.; CASTELLA, R.; LUNELLI, C. E.Produção de Poli (Ácido lático) a partir do soro de leite. The Journal of Engineering and Exact Sciences - JCEC. Vol. 03 N. 08, 2017.

FERNANDES, M. S. Enterococcus spp. e Bacillus cereus isolados do processamento de ricota: patogenicidade, formação de biofilmes multiespécie e detecção de autoindutores. 2014. 221f. Tese (Tecnologia de Alimentos) - Faculdade de Engenharia de Alimentos da Universidade Estadual de Campinas, Campinas - SP, 2014.

FERREIRA, L. R. Bebida isotônica formulada a base de permeado obtido da ultrafiltração do soro de leite adicionado de carotenóides em pó de pequi (caryocar brasiliense Camb.) 2017. 86f. Dissertação (Mestrado em Ciência e Tecnologia de Alimentos) - Universidade Federal de Viçosa, Minas Gerais - Brasil, 2017.

GUATEMIM, E. L. X.; SILVEIRA, S. M.; MILLEZI, A. F.; FERENZ, M.; et al.Evaluation of the microbiological quality of ricotta cheese commercialized in Santa Catarina, Brazil, Food Science and Technology, Campinas, v. 36, n4 4, p. 612-615, Oct.-Dec. 2016.

GUO, J.; WU, H.; DU, L..; ZHANG, W.; YANG, and. J. Comparative Antioxidant Properties of Some Gingerols and Shogaols, and the Relationship of Their Contents with the Antioxidant Potencies of Fresh and Dried Ginger (Zingiber officinale Roscoe). J. Agr. Sci. Tech, China, Vol. 16: p.1063-1072, 2014.

GRASSO, E. C.; AOYAMA, E. M.; FURLAN, M. R. Ação Antiinflamatória de Curcuma longa L. (zingiberaceae). Revista Eletrônica Thesis, São Paulo, ano XIV, n.28, p.117-129, $2^{\circ}$ semestre, 2017.

HAFEMANN, S. P. G.; AVANÇO, G. B.; SCAPIM, M. R. S.; ANTIGO, J. L.; et al.Ricota com Adição de Óleo Essencial de Orégano (Origanum vulgare Linneus): Avaliação Físico-Química, Sensorial e Microbiológica. Revista Brasileira de Produtos Agroindustriais, Campina Grande, v.17, n.3, p.317-323, 2015. 
HANNA, A. C. S. Aproveitamento de soro de queijo para a produção de pasta de ricota sabor tucumã (astrocaryum aculeatum meyer). 2015. 46f. Dissertação (Ciência dos Alimentos) Universidade Federal do Amazonas, Manaus, 2015.

HENING, B.; PIOLA, R. L. Desenvolvimento de ricota saborizada adicionada de oligofrutose. 2013. 47f. Monografia (Graduação em Tecnologia em Alimentos) Universidade Tecnológica Federal do Paraná - FTFP, Francisco Beltrão, 2013.

JAHN, R. C.; GARCIA, M. V.; COPETTI, M. V. Deterioração fúngica em indústria de queijo tipo tropical. Brazilian Journal of Food Research, Campo Mourão, v. 8, n. 1, p. 16-25, jan./mar. 2017.

LIMA, A.; SILVA, L. S.; CAVALCANTI, N. A. A.; CAMPOS, T. F. G. M. Gengibre (zingiber officinale roscoe), propriedades bioativas e seu possível efeito no diabetes tipo 2: estudo de revisão. Revista Saúde em Foco, Teresina, v. 1, n. 2, p. 15-25, ago/dez. 2014.

LIMA, B. B.; LEAL, M. C. Parâmetros indicadores de qualidade de queijos artesanais comercializados em Castro-PR. 2017. 30f. Monografia (Graduação em Tecnologia em Alimentos) - Universidade Tecnológica Federal do Paraná UTFP, Ponta Grossa, 2017.

MACHADO, N. A. F. Desenvolvimento e análise sensorial de bolo enriquecido com soro de leite e microencapsulado de própolis vermelha. 2017. 62f. Dissertação (Mestrado em Nutrição) - Universidade Federal de Alagoas, Maceió, 2017.

MAGENIS, R. B. Avaliação das propriedades físico-químicas, reológicas e do perfil eletroforético por SDS-PAGE de queijo minas frescal. 2015. 116f. Tese (Doutorado em Ciência dos Alimentos) - Centro de Ciências Agrárias da Universidade Federal de Santa Catarina - UFSC, Florianópolis, 2015.

MANCINI, S.; SECCI, G.; PREZIUSO, G.; PARISI, G.; PACI, GISELLA. Ginger (Zingiber officinale Roscoe) powder as dietary supplementation in rabbit: life performances, carcass characteristics and meat quality. Italian Journal of Animal Science, p. 1-6, jan. 2018.

MARCHI, J. P.; et al. Curcuma Longa L., o Açafrão Da Terra, e seus benefícios medicinais. Arquivos de Ciências da Saúde da UNIPAR, Umuarama, v. 20, n. 3, p. 189-194, set./dez. 2016.

MARIOT, A.; GIBIKOSKI, C. Z.; VIEIRA, R. M.; VILARINS, V. Produção de ácido lático a partir de soro de leite.2017. 293f. Projeto de pesquisa (Graduação em Engenharia Química) - Universidade do Sul de Santa Catarina - UNISUL, Tubarão - SC, 2017.

MARMITT, D. J.; REMPEL, C.; GOETTERT, M. I.; SILVA, A.D. Análise da produção científica do Curcuma longa L.(açafrão) em três bases de dados após a criação da RENISUS. Revista Pan-Amazônica de Saúde, Lagedo - Rio Grande do Sul, Brasil, v. 7, n. 1, p. 71-77, 2016. 
MARQUES, L. F.; COSTA, T. L., MOURA, R. L., MARIA, A.; COSTA, N. M., CHAVES, C. G.; FREITAS, R. M. Adição de orégano na produção de ricota: um teste de aceitação. Anais: JORNADA NACIONAL DA AGROINDÚSTRIA, Bananeiras: UFPB, 2008.

MEDEIROS, R. O. N. B. Estudo da aplicação na área da saúde do gengibre, sua caracterização química. 2017. Dissertação (Mestrado em Ciências Farmacêuticas)Instituto Superior de Ciências da Saúde - Egas Moniz, Nov. 2017.

MEIRA, Q. G. S. Produção e caracterização de Ricota Caprina adicionada de bactérias Probióticas. 2015.140f. Tese (Doutorado em Ciência e Tecnologia de Alimentos), Universidade Federal da Paraíba - UFPB, João Pessoa, 2015.

MENDES, F. S.; SOUZA, C. F. V. Qualidade microbiológica de soro de queijo e soro de ricota de laticínios do vale do Taquari, Rio Grande do Sul. Revista Brasileira de Tecnologia Agroindustrial, Ponta Grossa, v. 11, n. 2, p. 2437-2450, jul/dez. 2017.

NASCIMENTO, J. C. S.; LIMA, M. S.; ALBUQUERQUEK. G. S.; CHINELATE, G. C.B.; SILVA, J. J. C. Avaliação da ação de diferentes antimicrobianos naturais contra microrganismos patogênicos. Revista Brasileira de Agrotecnologia, Pernambuco, v. 7 , n. 2, p.203-208, 2017.

NOGUEIRA, A. M. S.; STELLA FLÁVIA RENZETI MARIOTTI, S. F. R.; BUENO, S.M. Análise da qualidade microbiológica De queijo ralado comercializados em São José do Rio Preto - SP. Revista Cientifica UNILAGO, p. 129-135, 2013.

Norman, J. 2004. Ervas aromáticas e especiarias, Editora Civilização, Porto.

OLIVEIRA, J. Uso de critérios para avaliação da qualidade microbiológica de um laticínio. 2016. 53f. Monografia (Bacharel em Engenharia de Alimentos), Universidade Federal da Fronteira Sul - UFFS, Laranjeiras do Sul, 2016.

OLIVEIRA, J. O.; VILELA, L. T. O .; SILVA, L. H. O.; NASCIMENTO, T. S.; et al.Análise microbiológica de especiarias desidratadas comercializadas em feiras livres de Cuiabá, Mato Grosso. Journal Health Npeps, Cuiabá - Mato Grosso, Brasil, v. 2, n. 2, p. 365-379, 2017.

OLIVEIRA, M. B. Avaliação de queijos ricota comercializados em Goiânia-GO e queijos processados com diferentes concentrações de leite e adicionados de proteínas de soja e cálcio. 2012. 109f. Dissertação (Mestrado) - Escola de Agronomia e Engenharia de Alimentos, Universidade Federal de Goiás - UFG, Goiânia, 2012.

PREIS, C.; C.; CHAGAS, T. E. R.; RIGO, E. Avaliação da ação antimicrobiana de óleo essencial de orégano (origanum vulgare) em ricotas adicionadas de especiarias. Revista do Congresso Sul Brasileiro de Engenharia de Alimentos. v. 1. n. 1, p. 1-8, 2015.

ALES, W. B.; CAVEIÃO, C.; VALENTIM, D.; CASTRO, L. Análise microbiológica em ricotas frescal no município de Curitiba - PR. Revista de Biotecnologia \&

Ciência. Vol.5, Nº1, p.16-24, 2016. 
SANTOS, P. D. F. Avaliação da atividade antimicrobiana de microcristais de curcumina. 2015. 36f. Monografia (Bacharel em Engenharia dos Alimentos) Departamento de Alimentos- DALIM - da Universidade Tecnológica Federal do Paraná - UTFPR, Paraná, 2015.

SANTOS, R. R.; SOUZA, A. L. R.; TROMBETE, F. M.; MELO, N. R. Proteína do soro de leite: Aproveitamento e aplicações na produção de embalagem biodegradável. Revista Verde de Agroecologia e Desenvolvimento Sustentável, Pombal - PB, Vol. 10. $n^{\circ} 5$, p. 51 - 58, Dez., 2015.

SANTOS, S.; et al. Características tecnológicas, de qualidade e potencialidades da cadeia produtiva de queijo colonial na região Sul do Brasil: uma revisão. FTT Journal of Engineering and Business, São Bernardo do Capo - SP, v. 1, n. 2, p. 50-64, fev/jun, 2017.

SATTIN, E.; ANDREANI, N. A.; CARRARO, L.; FASOLATO, L.; et al. Microbial dynamics during shelf-life of industrial Ricotta cheese and identification of a Bacillus strain as a cause of a pink discolouration. Food Microbiology, vol. 57, p. 8-15, dez. 2016.

SEDLMAIER, A. W.; SANTOS, A. C.; PERES, A. P. Avaliação sensorial de carne moída acrescida de condimentos naturais com baixo teor de sódio. Cadernos da Escola de Saúde - Faculdades Integradas do Brasil, Curitiba, v. 1, n. 11, p. 90-102. 2017.

SHIBATA, L. W. Análise físico-química, microbiológica e sensorial do queijo de coalho condimentado produzido a partir do leite de cabra congelado. 2018. 32f. Monografia (Graduação em Zootecnia) - Centro de Ciências Agrárias. Universidade Federal da Paraíba - UFPB, Areia - PB, 2018.

SILVA, E. A.; SANTOS, E. A. L.; AQUINO, A. B.; ARAUJO, J. M.; et al. Processamento de ricota natural e condimentada: avaliação microbiológica e sensorial. Revista GEINTEC - Gestão, Inovação e Tecnologias, São Cristovão/SE, v. 4, n. 2, p. 788-795, 2014.

SPANU, C.; PIRAS, F.; MOCCI, A. M.; NIEDDU, G.; SANTIS, E. P. L.; SCARANO, C. Use of Carnobacterium spp protective culture in MAP packed Ricotta fresca cheese to control Pseudomonas spp. Food Microbiology, 74, p.50-56, 28 fev. 2018.

SOUSA, J. P. B.; et al. Açafrão em pó (Curcuma longa L.) em dietas de frangos de corte. In: Colloquium Agrariae.v. 13, n.2, Mai-Ago. 2017, p.97-108, 2017.

SOUZA, M. Y. M. Análise de creme de Ricota: Caracterização físico-química e classificação quanto ao teor de gordura no extrato seco. 2014. 29f. 21. Ed. Monografia (Graduação em Química Industrial) - Universidade Estadual da Paraíba, Centro de Ciências e Tecnologia, Campina Grande - PB, 2014.

TAVARES, F. O.; PIERETTI, G. G.; ANTIGO, J. L.; POZZA, M. S. S.; SCAPIM, M. R. S.; et al. Cobertura comestível adicionada de óleos essenciais de orégano e alecrim para uso em ricota. Rev. Inst. Laticínios Cândido Tostes, Juiz de Fora, v. 69, n. 4, p. 
249-257, jul./ago., 2014.

TOZETTO, L. M. Produção e caracterização de cerveja artesanal adicionada de gengibre (Zingiber officinale). 2017. 80f. $30 \mathrm{~cm}$. Dissertação (Mestrado em Engenharia de Produção) - Universidade Tecnológica Federal do Paraná - UTFP, Ponta Grossa, 2017. 